\title{
Improving Tourist Attractions Revisit Rate: The Perspective of Network Marketing SURE Model
}

\author{
Yanhong Liu, 1,2,a, Yuan Le ${ }^{1, b}$ and Xiaowen $\mathrm{Jie}^{2, \mathrm{c}^{*}}$ \\ ${ }^{1}$ Tourism and Culture Department, Normal College, Shenzhen University, China \\ ${ }^{2}$ Strategy and HR Management Department,Business School, Sichuan University, China \\ a841226273@qq.com, b379915936@qq.com, cjiexw@vip.163.com \\ ${ }^{*}$ Corresponding author
}

Keywords: Network marketing, SURE model, Revisit rate, Tourist attractions.

\begin{abstract}
With rapid development of tourism, revisit rate in scenic spots becomes one of the important indicators to measure the vitality of tourist attractions. Increasing revisit rate can effectively enhance the sustainable development ability of tourism enterprise. Network marketing SURE model follows the diversity of media, new generation of consumers, fragmented entertainment time as its insight of Spreading, Unification, Route and Exactness(SURE). Questionnaires and cases collection are conducted to combine the characteristics of SURE model with the operating model of scenic spot to explore the feasibility of improving revisit rate. It is conducive that improving revisit rate of tourist attractions constitutes new generation of young consumers. A set of scenic closed loop marketing is found which provides corresponding strategies for scientific and sustainable development of the various stages.
\end{abstract}

\section{Introduction}

In order to meet needs of consumers with rapid development of market economy in China, product positioning of tourism industry has gradually shifted from overall development of "big and full-scale" to "small and refined"niche market. China's tourism revenue reached $\$ 4.69$ billion in 2016, up $13.6 \%$ from a year earlier ${ }^{[1]}$. Tourist attractions revisit rate can bring increasing travel revenue, but growth of overall income does not represent benign development environment. $70 \%$ scenic spots companies in China operated at a loss, $20 \%$ maintained unprofitable, and $10 \% \mathrm{kept}$ profitable (Dong,2010) ${ }^{[2]}$. A survey of revisit rate (Dong,2017) showed that most foreign sites had a higher rate of revisit: Tokyo's Disneyland Resort rate was $83.6 \%$, while the highest rate of revisit in China was 28.3\% including Shenzhen Happy Valley and other theme parks, with revisit rate for most China tourist attractions being low to $1 \%{ }^{[3]}$.

The main factors affecting tourist revisit are the image perception of tourist destinations and the evaluation of the services that tourists enjoy in the first experience (Gyte,1989) ${ }^{[4]}$. Risk aversion, emotional attachment and social needs are found on the basis of data analysis as main factors affecting the willingness of tourists to revisit their activities (Backman and Crompton,1991) ${ }^{[5]}$. Formica and Uysal (1989) improve the way that tourist image is perceived to emotional attachment of consumers ${ }^{[6]}$.

High revisit rate is an important indicator of the vitality of a tourist area and the guarantee of stable profit revenue. Revisit motivations focus on new project, relatives and friends invitation and revisit discount(Zong and Yao,2014) ${ }^{[7]}$. However, the development of new projects is not only a long period, but also a large cost investment.

There are several directions for how to improve revisit rate, but the first thing needs to make clear is the target customer. In the white paper released by Tencent Data Lab in 2017, tourists under the age of 30 account for $68 \%$ of the total. Those young people being born after 2000 and 1990 have become new generation of tourist, affecting the direction of the market. Therefore, it is more scientific to explore the new generation of tourists as main consumers. Internet users under age of 30 in China accounts for $83 \%$, whose consumption being guided by the Internet. 
Undoubtedly, the study of revisit rate is an important way of guiding tourist attractions to the scientific development, having powerful effect on the profit from new generation of consumption in the near future. This study discusses the feasibility and strategy of the SURE model of the network marketing so as to attract internet users in promotion designing.

\section{Literature review}

\subsection{SURE model of network marketing}

SURE model is the theoretical framework of new birth of online marketing in recent years. Compared with the 4P theory of traditional marketing and the market STP positioning theory, it is more in line with the characteristics of network marketing, fragmentation, precision and social characteristics. SURE model is the English abbreviation of spreading, unification, route and exactness(Xie,2015) ${ }^{[8]}$.

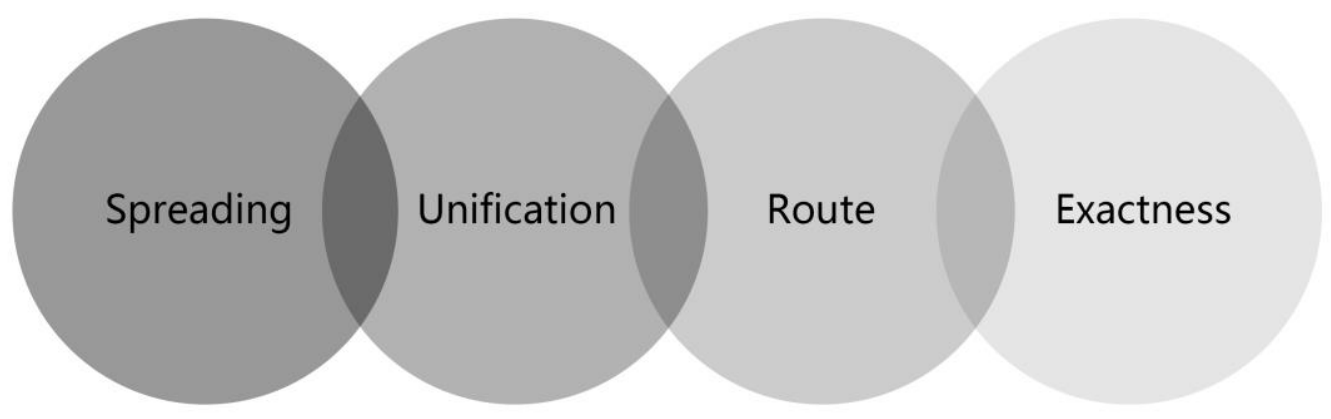

Fig 1. SURE model of network marketing-adapted from "Internet marketing” (Xie,2015)

The advent of internet is a sign of the "weak authority, strong social" media system, while internet in the 21 st century has led to decline of traditional media.As Fig 1 shown, "Spreading"and "Word of mouth" have become credible source of information for consumers. "Unification" is reflected in the upgrade of business and consumer relations, from traditional "business relationships" to "knowledge sharing" or "stakeholder"(Scott,2010) ${ }^{[9]}$. In traditional media technology, the relationship between consumers and advertising marketing is a one-way passive acceptance which consumers accept information as long as the media releases information ${ }^{[10]}$. "Route" of SURE model promotes the result of capturing consumer searches through masquerading as knowledge content for soft advertising by internet entry, search engine and node. "Exactness" of SURE model recommends that the product features be tested after the suitability of the consumer personality, network medium be chosen for target consumers and good advertising be produced by word of mouth spread among consumers.

\subsection{Revisit rate}

In Wikipedia, the definition of "revisit" means that tourists visit their visited destinations again.Some scholars believe that the "early wanderer" and "repeat visitor" are the basic components of travel activists, and any tourists are made up of these two kinds of people(Um,Chon and Ro, 2006) ${ }^{[11]}$. Revisit rate represents the willingness of tourists to buy in the view area for a period of time, also shows the level of satisfaction in the tourist area.(Yang and Miao,2009) [12]. The design and development of a new tourist scenic area requires not only huge investment, but also heavy sale. Increase of revisit rate can guarantee stable tourist source and bring long-term benefit while tourists enjoy their travel through interviews with other tourists.(Gitelson and Crompton,1983) ${ }^{[13]}$. 


\subsection{Place attachment}

Place attachment refers to the special attachment that occurs between people and places because of special interaction and emotional communication.Visitors' degree of attachment can reflect the extent to which visitors are satisfied with their tourist destinations, so they can be measured as one of the indicators of quality of tourist attraction(Yang,2015) ${ }^{[14]}$. The study of place attachment is conducive to improvement of the revisit rate. In SURE model, place attachment can be seen as a high degree of fan fusion between scenic spots and tourists as the source of "word of mouth spread" .

\section{Methodology and data Analysis}

This study focuses on the feasibility of improving revisit rate based on SURE model in internet era. "Social attributes" and strength of tourism social topics are related to the validity of the word of mouth spread strategy with SURE model. In the questionnaire, "word of mouth" and "social attributes" are added to the influence of the tourists' revisit rate. The source of tourist information can be used to show that internet end information entry is the main source of information collection in SURE model. Therefore, the questionnaire designs the relevant problems for the source of new network and new side of the tourist information to get data collection. We have offered on line and off line questionnaires and collected them from March to April, 2018. According to the statistical data, the total amount of the questionnaires are 258, valid questionnaires are 241 , and effective rate is $93.4 \%$.

\subsection{Analysis about "spreading"}

From the data in questionnaire, $64.1 \%$ of the tourists with revisiting plans are motivated by "good experience brought by the last tour". It can be seen that the psychological cognition of the initial tourism in minds of tourists on the tourist attractions will become important basis for the tourists to make a revisit again. Factors such as the service project designed by the tourist destination, publicity effect of the tourist attraction and the gap between the real service experienced by tourists will not only affect the tourists' evaluation of this tour, but also directly impact the possibility of second visit.

According to the survey, $96.34 \%$ of revisit tourists are willing to share their travel experiences with others, while $40.3 \%$ of the tourists get information mainly from "family or friends who have been there"before their travel. Among the questions about whether the evaluation of tourist reputation will affect the selection of tourist destinations, $45.3 \%$ of tourists will choose not to go to the scenic spot because of the vicious evaluation of the tourist attraction by public opinion. It can be seen that tourism experience will be more likely to be the basis for other tourists to judge their scenic spot.

To sum up, "word of mouth spreading" has great influence on the travel choice of the two groups of tourists - "beginner" and "re-traveler". It also indicates that, as the main provider of service projects in tourism activities, scenic spots need to ensure the quality of the service that they will offer or have provided, and the service that can be reasonably matched with the publicity effect, so as to control public opinion and praise of tourists.

\subsection{Analysis about "unification"}

According to the survey, $53.9 \%$ of the tourists choose to obtain relevant information of the tourist destination through tourism new media (We Chat, Weibo, etc.). In internet era, the cultivation of tourists' impression on the scenic spot starts before the tourists arrive at the scenic spot. Scenic spots can inject good first impression to the tourists through the operation and maintenance of new media portal.

New media can be used to enhance relationship and integration with tourists, which can make tourists have "local attachment" to scenic area, thus improving revisit rate. Studies have shown that when tourists have emotional connection with certain tourist area, they will have an "attachment to the place", which will affect their satisfaction with the place and their consumption behavior in the 
place. Therefore, increasing the frequency of online fan interaction, allowing tourists to participate in the construction of the scenic area to realize "fan democracy", creating "local attachment", and promoting improvement of revisit rate.

\subsection{Analysis about "route"}

According to the survey results, $76.3 \%$ of the tourists said they would not actively search for scenic spots after the initial visit. Thus, the control of search results in path marketing only serves as means to attract new visitors. To improve revisit rate of scenic spots, it is still necessary to carry out long-term operation of loyal tourists by combining the ideas of "spreading" and "unification", so as to maintain long-term attention of scenic spots.

\subsection{Analysis about "exactness"}

According to the survey data, different tourists have different requirements for service projects in tourist attractions, with $56.9 \%$ of the tourists paying more attention to food service in the process of tourism, while $65.4 \%$ being focus on traffic factors.

Meanwhile, accurate positioning of service projects in scenic spots can improve the correlation between some tourism-related keywords and scenic spots. In the questionnaire survey, $83.8 \%$ respondents would associate "beach" with "Sanya" when asked about their impressions of domestic tourist attractions, 79.6\% respondents associate "snow and ice" with "Harbin". 62.2\% visitors associate "grassland" with "Inner Mongolia". Therefore, precision marketing can effectively attract tourists who are in need of specific tourism scenes or tourism service projects, and the tourist intention of these tourists will be more intense. Keywords identified in precision marketing can also serve as the path entrance to guide tourists to discover search advertisements in path marketing, so as to increase their attention and improve tourists' revisit rate.

\section{Summary}

As literature review and questionnaire analysis shown, factors affecting tourists' revisit rate mainly include tourists' attention to the tourist destination, their satisfaction with the initial travel, their overall public praise of the tourist destination and their attachment to the site.

We can see from Fig 2 that SURE model is an integral marketing closed-loop approach.Its main impact paths on tourists are "Draw public attention- increase place attachment - constitute the initial tour - become a fan and spread - promote the repeat tour", so as to attract other first-time visitors' attention and continue the process. So SURE model has a certain role in improving revisit rate, and can maintain high attention from the initial tour to the re-tour of visitors.

SURE model has some limitations because that its every step has very strong correlation. Improving revisit rates is a longer-term approach, effective results are difficult to achieve in the short time. However, it can help foster potential "fans" of scenic spots, form "the traveller seeds", finally achieve long-term sustainable development. 


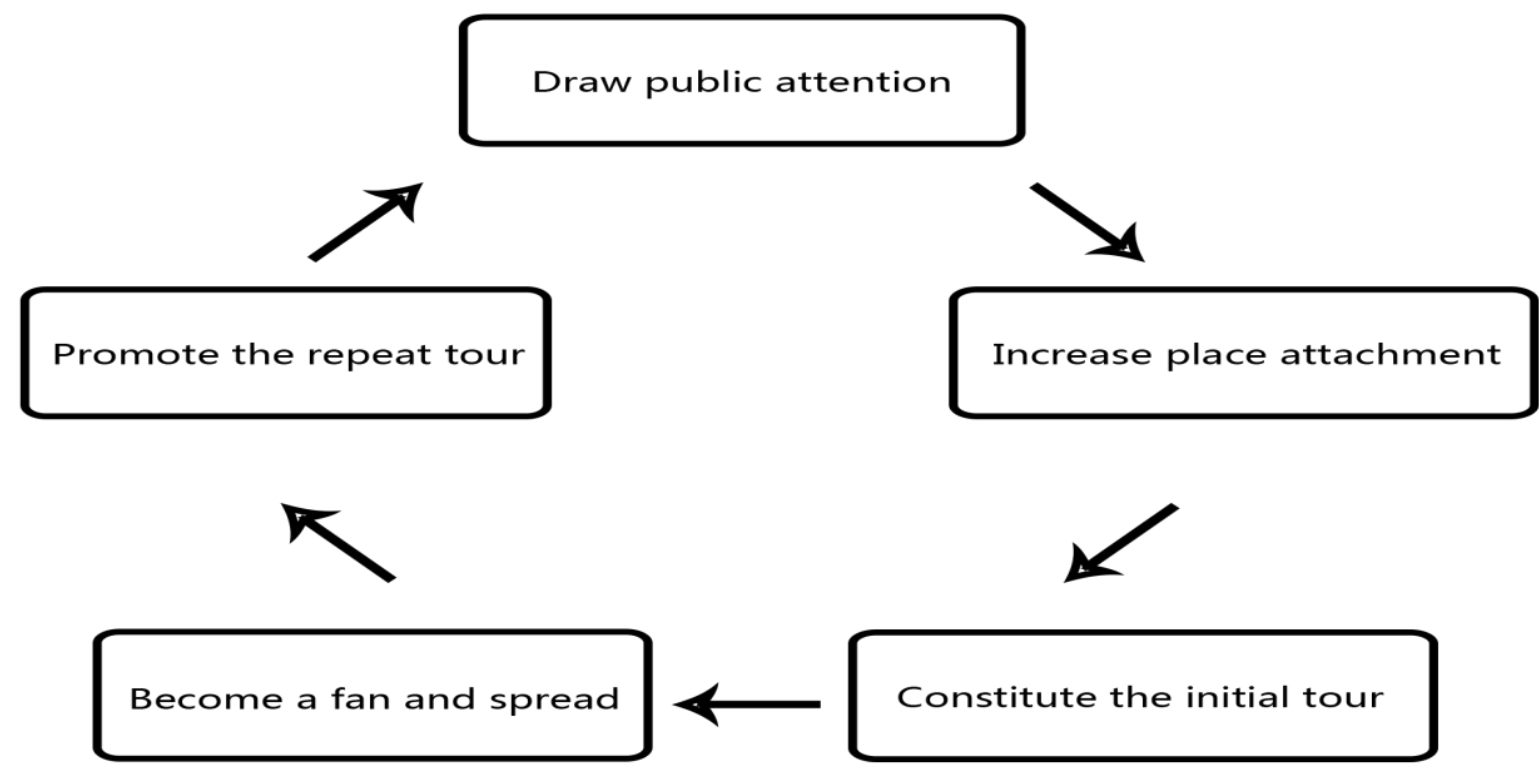

Fig 2. SURE model of integral marketing closed-loop approach (made by author)

\section{Acknowledgement}

This research was financially supported by Finance Department Foundation of Guangdong (Grant NO.Z201827), Guangdong Higher Education Teaching Reform Project Foundation(Grant NO.201627334) and Tourism Creative Planning Competition Funds for University students(Grant NO.2018261007) .

\section{References}

[1] C.Wu andY.Yuan. China's tourism revenue reached $\$ 4.69$ billion in 2016, up 13.6\% from a year earlier[EB/OL].http://news.ifeng.com/a/20171108/53084039_0.shtml.

[2] G.Z.Dong.Theme parks: urban commercial clusters and cultural games-interpreting the development course and strategic trends[J].Modern City Research, vol. 3, pp. 7-13, 2010.

[3] X.S.Zhang.Revisit rate for most tourist attractions in China being low to $1 \%$ [EB/OL].http://epaper.bjnews.com.cn/html/2017-06/28/content_686555.htm?div=1.

[4] D.M.Gyte and A. Phelps. Patterns of Destination Repeat Business: British Tourists in Mallorca, Spain [J].Journal of Travel Research, vol. Summer, pp. 24-28, 1989.

[5] S.J.Backman and J.L.Crompton. The usefulness of selected variables for predicting activity loyalty [J].Leisure Study, vol. 13, 1991.

[6] S.Formica and M.Uysal.Market segmentation of an international cultural-historical event in Italy[J].Journal of Travel Research, vol. Spring, pp. 16-24, 1998.

[7] C.L.Zong and J.J.Yao.Research on improving tourist revisit rate-Take Wuhu Fantawild Cartoon Theme Park for example[J].Journal of Hunan University of Technology Social Science Edition, vol. 19, pp. 49-52, 2014. 
[8] D.Xie. Internet marketing: the subversion and transformation of ideas[M].Beijing: Mechanical Industry Press, pp. 29-60, 2015.

[9] D.M.Scott.Word-of-mouth marketing[M].Beijing: Posts and Telecom Press, pp. 32-33, 2010.

[10]W.J.Severin and J.Tankard.Communication Theory[M].Beijing:Communication University of China,pp.45-48,2006.

[11]S.Um,K.Chon and Y.R.Antecedents of Revisit Intention[J].Annals of Tourism Research, vol. 33, pp. 1141-1158, 2006.

[12]A.H.Yang and C.C.Miao. Economics of tourism[M].Beijing:Tsinghua University Press,pp.108-109,2009.

[13]R.J.Gitelson and J.L.Crompton. The planning horizons and sources of information used by pleasure vacationers[J]. Journal of Travel Research,vol.Winter, pp. 2-7, 1983.

[14]Y.Yang.A literature review of place attachment both at home and abroad[J]. Journal of the Graduate Sun Yat-sen University,vol.33, pp.26-37, 2011. 